\title{
Uncommon presentation of Pityriasis versicolor; hyper and hypopigmentation in a same patient with variable treatment response
}

\author{
Riti Acharya, Madhu Gyawalee
}

Lecturer, Patan Academy of Health Sciences, Lalitpur, Nepal

Corresponding author: Dr. Riti Acharya, E-mail: riti_acharya@hotmail.com

\begin{abstract}
Pityriasis versicolor (PV) is a superficial fungal infection of skin caused by yeast Malassezia. Typical lesions are multiple small, round macules that coalesce to form confluent lesions. Eruption varies in color from patient to patient, but each person's lesions are usually of a single hue. Here we present a combination of both hypo and hyperpigmentated lesions of PV in a single patient. On treating the patient for 4 weeks with antifungals, complete clearance of hyperpigmented lesions were seen whereas hypopigmented ones became more accentuated.
\end{abstract}

Key words: Hyperpigmentation; hypopigmentation; Pityriasis versicolor

\section{INTRODUCTION}

Pityriasis versicolor $(\mathrm{PV})$ is a superficial fungal infection of skin caused by yeast of genus Malassezia (notably Malassezia globosa, less commonly, M. Furfur and M. Sympodialis) [1]. Prevalence ranges from $1 \%$ in dry and temperate climates to $50 \%$ in tropics [2]. It occurs when the yeast form of the organism converts to the hyphal form. PV is characterized by multiple small, round macules that coalesce to form confluent lesions of varying colour.

\section{CASE REPORT}

23 year old lady presented with asymptomatic rash all over her upper trunk and upper extremities since 5 years. On examination, the lesions were symmetrically distributed hyperpigmented, round to oval macules with fine scales predominantly involving intertriginous areas (neck, axilla, inter and inframammary areas) (Fig. 1) whereas similar hypopigmented lesions were present over the back, chest and bilateral upper limb (Figs. 2a and 2b). To evaluate the color of the lesions, the patient was examined by Wood's lamp (Fig. 3). Yellow hue of the lesions confirmed PV which was more prominent in hyperpigmented lesions than hypopigmented ones. In direct $\mathrm{KOH}$ skin smear, fungal elements with characteristic 'spaghetti and meatball' pattern was seen. On treating the patient with ketoconazole $200 \mathrm{mg}$ daily for 7 days and cream ketoconazole twice daily for one month, the hyperpigmented lesions completely resolved with marked accentuation of hypopigmented ones over the previous sites (Figs. 4a and 4b).

\section{DISCUSSION}

Pityriasis versicolor is caused by dimorphic lipiddependent yeasts Malassezia spp. formerly known as Pityrosporum) which are normal human saprophytes. It is more common in the tropics than in temperate climates. Generally lesions are restricted to anatomical sites that are covered by clothing, suggesting the role of increased heat and moisture in the pathogenesis of the lesions. Some patients experience mild pruritus, but it is usually asymptomatic. As the name suggests, it may present with color ranging from pink to tan brown to black [2]. In general, hyperpigmented, red to brown lesions erupt in fair-skinned patients whereas

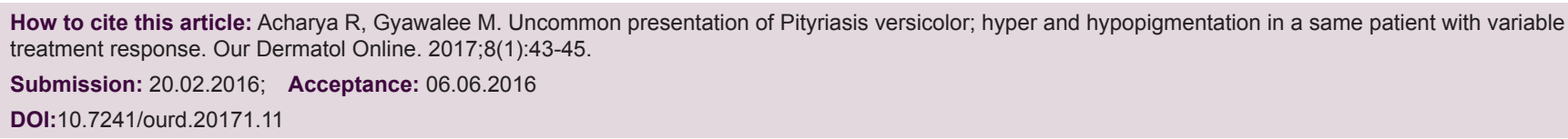




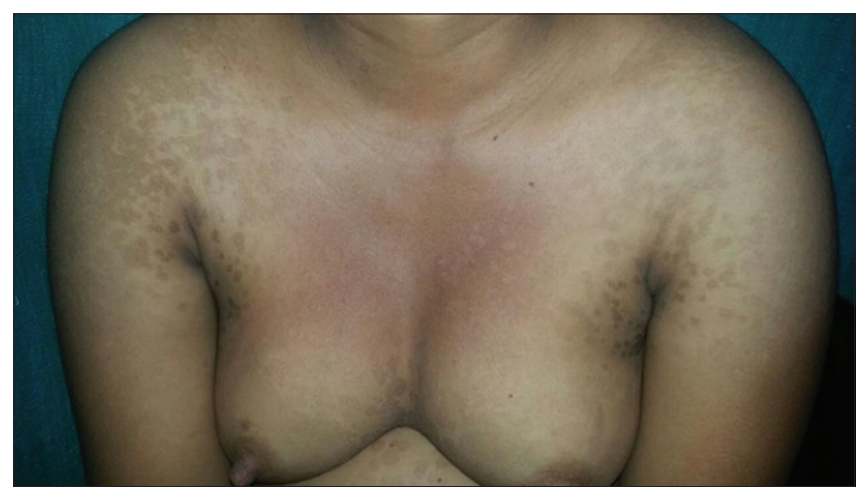

Figure 1: Hyperpigmented lesions of Pityriasis versicolor seen over $\mathrm{B} / \mathrm{L}$ axilla extending to chest and arms, inter and infra mammary area.

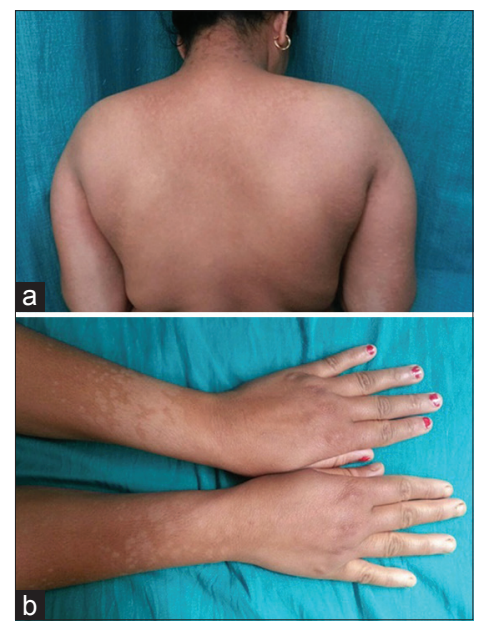

Figure 2 : ( $a$ and $b$ ) Hypopigmented macules of Pityriasis versicolor over upper back, arms and forearm.

those with dark skin tend to have hypopigmented lesions [3].

Skin pigmentary changes of PV can occur as either hyperpigmented or hypopigmented lesions in accordance with interactions between Malassezia yeasts and skin characteristics, such as lipoperoxidation process, stimulus of inflammatory cell to melanocytes, and increased thickness of keratin layer. However, the precise factors that enhance susceptibility to Malassezia yeasts and provoke PV is yet to be defined [4].

In hypopigmented lesions, the interaction between skin barrier components and Malassezia yeasts, such as lipoperoxidation process cause cytotoxic effect to make hypopigmented patches. To add, production of dicarboxylic acid which inhibits tyrosinase which is essential for skin pigmentation, inhihibition of tanning due to overlying scales, or abnormally small melanosomes are other factors causing hypopigmentation. Whereas, the hyperpigmented lesions occur due to thicker

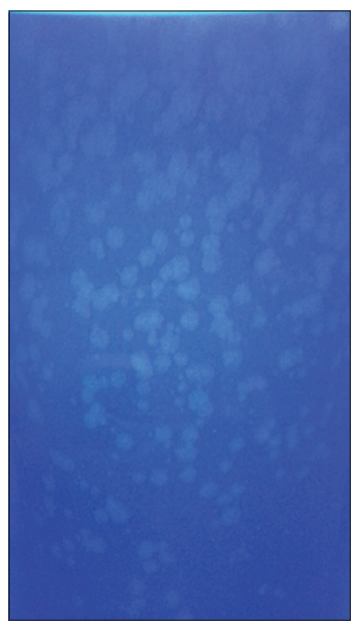

Figure 3: Wood's lamp examination showing accentuation of lesions of hyperpigmented (A) compared to hypopigmented (B) P versicolor.



Figure 4: $(a$ and b) Four weeks after treatment showing complete clearance of hyperpigmented macules with accentuation of hypopigmented macules.

stratum corneum, larger melanocytes and inflammatory reactions against fungus. Hence hyperpigmentation could occur as a result of both inflammation and increased melanin production $[4,5]$.

Diagnosis is usually by clinical examination, $\mathrm{KOH}$ examination shows typical "spaghetti and meat ball" appearance, and Wood's lamp examination shows orange to yellow fluorescence. Identification by culture requires lipid enrichment of the media and is rarely done to establish the diagnosis. It can be confirmed by histopathology which shows yeasts in the stratum corneum and sometimes in the perifollicular region.

PV is difficult to cure, as relapse following treatment can be as high as $80 \%$ within 2 years. Topical antifungals are currently the first line of treatment and systemic antifungals are recommended for severe or recalcitrant 
cases. Among systemic antifungal, ketoconazole was once the gold standard for oral treatment. Currently, itraconazole, fluconazole, and pramiconazole are effective antifungals [6]. Patients of PV should be counselled that hyper- or hypopigmentation may persist and may take months to recover normal skin appearance despite successful treatment.

In our case, rapid control of inflammation could have cause the hyperpigmented lesion of PV to regain normal pigmentation quickly. But decrease in the activity of tyrosinase caused by dicarboxylic acids, produced by the organism and direct cytotoxic effect on melanocytes in hypopigmented lesions might have taken longer time to recover and regain normal pigmentation.

\section{CONCLUSION}

PV is common superficial fungal infection of skin causing pigmentary changes. Cosmetic concern often warrants treatment. However, even after successful treatment pigmentary alteration may persist. Hyperpigmented lesions resolve early than hypopigmented ones which could be due to faster resolution of ongoing inflammation in hyperpigmented lesion with more gradual recovery of tyrosinase activity and cytotoxic effects of melanocytes on hypopigmented lesions.

\section{REFERENCES}

1. Lyakhovitsky A, Shemer A, Amichai B. Molecular analysis of Malassezia species isolated from Israeli patients with pityriasisversicolor. Int J Dermatol. 2013;52:231-3.

2. Gupta AK, Bluhm R, Summerbell R. Pityriasis versicolor. JEADV. 2002;16:19-33.

3. Leung AK. Pityriasis versicolor. In: Lang F, ed. The Encyclopedia of Molecular Mechanisms of Disease. Berlin: Springer-Verlag, 2009;1652-4.

4. Park HJ, Lee YW, Choe YB, Ahn KJ. Skin Characteristics in Patients with Pityriasis Versicolor Using Non-Invasive Method, MPA5. Ann Dermatol. 2012;24:444-52.

5. Zonunsanga. Atypical pityriasis versicolor: case report. Our Dermatol Online. 2015;6:198-200.

6. Gupta AK, Foley KA. Antifungal Treatment for Pityriasis Versicolor. J Fungi. 2015;1:13-29.

Copyright by Riti Acharya, et al. This is an open access article distributed under the terms of the Creative Commons Attribution License, which permits unrestricted use, distribution, and reproduction in any medium, provided the original author and source are credited.

Source of Support: Nil, Conflict of Interest: None declared. 